\title{
Correction: Hydraulic tomography analysis of municipal-well operation data with geology-based groundwater models
}

\author{
Xin Tong ${ }^{1} \cdot$ Walter A. Illman ${ }^{1} \cdot$ Steven J. Berg ${ }^{1,2} \cdot$ Ning Luo $^{1}$ \\ Published online: 6 May 2021 \\ (C) Springer-Verlag GmbH Germany, part of Springer Nature 2021
}

\section{Correction: Hydrogeology Journal https://doi.org/10.1007/s10040-021-02320-4}

The original version of this article unfortunately contained a mistake.

1) Missing characters ' $K$ ', ' $T$ ' and ' $S$ ' are found in sections "Introduction", "Description of groundwater models", and "Model validation results".

Section "Introduction", third paragraph should read:

The complexity and susceptibility of the Waterloo Moraine to overexploitation of groundwater resources and its potential contamination requires a sound understanding of local hydrogeology, including the reliable estimation of hydraulic parameters such as hydraulic conductivity $(K)$ and specific storage $\left(S_{\mathrm{s}}\right)$.

Section "Introduction", sixth and seventh paragraphs should read:

In a previous study by Luo and Illman (2016), long-term pumping/injection events and water-level variation records were used to estimate hydraulic parameters including transmissivity $(T)$ and storativity $(S)$ for the shallow aquifer (AFB2) within the

The online version of the original article can be found at https://oi.org/ $10.1007 / \mathrm{s} 10040-021-02320-4$

Xin Tong

x23tong@gmail.com

Walter A. Illman

willman@uwaterloo.ca

Steven J. Berg

sberg@aquanty.com

Ning Luo

nluo1222@gmail.com

1 Department of Earth \& Environmental Sciences, University of Waterloo, 200 University Avenue West, Waterloo, Ontario N2L 3G1, Canada

2 Aquanty Inc, 564 Weber St N., Unit 2, Waterloo, Ontario N2L 5C6, Canada
Waterloo Moraine. A set of $T$ and $S$ values were estimated between each production and monitoring borehole by fingerprinting the water-level variations to pumping/injection rate changes. The fingerprinting process was accomplished through the Theis (1935) model implemented in the WELLS code (Mishra and Vesselinov 2011) coupled with a nonlinear parameter estimation code PEST (Doherty 2005). The Luo and Illman (2016) study showed that long-term municipal water-level records were amenable for hydraulic parameter estimation, as the geometric means of the individual $T$ and $S$ estimates were similar to previous pumping test results at the study site. However, the wide range of estimated $T(9-55,335 \mathrm{~m} /$ day $)$ and $S(0.002-0.736)$ indicated the high degree of heterogeneity of the investigated aquifer. Furthermore, poor validation results using data that were not used for calibration purposes suggested that $T$ and $S$ estimates from individual pumping and monitoring boreholes may not be suitable for the drawdown prediction of other monitoring wells. In order to increase the accuracy of parameter estimates at this site, Luo and Illman (2016) concluded that a more sophisticated groundwater flowmodel that considers heterogeneity in $K$ and $S_{\mathrm{s}}$, as well as better accounting of the forcing functions (i.e., initial and boundary conditions as well as source/sink terms), is needed to obtain more accurate parameter estimates.

There are a number of approaches to map $K$ heterogeneity. A conceptually simple approach is to map the $K$ heterogeneity through the interpolation of small-scale $K$ estimates including permeameter tests, slug tests and single-hole tests, but a large amount of data is required. For example, Rehfeldt et al. (1992) estimated that about $400,000 \mathrm{~K}$ measurements would be required to accurately predict the transport of tracers in an alluvial aquifer at the Columbus Air Force Base in Mississippi, USA, commonly known as the Macrodispersion Experiment (MADE) site. Thus, it would be prohibitively expensive and timeconsuming to perform such analyses at a municipal wellfield.

Section "Introduction", tenth paragraph should read:

Previous studies conducted in the study area have mostly relied on pumping tests to estimate hydraulic parameters of various units (e.g., CH2M HILL 2003a, b; GolderAssociates 2011; 
Matrix Solutions Inc, S.S. Papadopulos and Associates 2014a, b), but such tests typically require shutting down the municipal wells and the interpretation is more difficult when the municipal wells are in operation. In order to overcome this difficulty and apply HT analysis without conducting sequential pumping tests, long-term pumping/injection events and water-level variation records obtained during municipal well field operation are used in this study to jointly calibrate a groundwater flow model consisting of homogeneous geological units to estimate the $K$ and $S_{\mathrm{s}}$. As an initial attempt, geological models are used for HT analysis in this study, as previous studies have shown the importance of geological data in parameter estimation when wells are far apart (Illman et al. 2015; Zhao et al. 2016; Luo et al. 2017; Zhao and Illman 2017, 2018). However, since perfect knowledge of stratigraphy is not available, there is a critical need to assess the impact of various conceptualizations of site geology on groundwater model calibration and HT at the field scale.

Section "Introduction", 12th paragraph should read:

The overall goal of this study is to examine the feasibility of conducting HT analysis of existing water level records influenced through municipal well field operations. Specifically, the objectives of the study are to: (1) demonstrate the usefulness of long-term pumping/injection and monitoring well records obtained through municipal well field operations for estimating hydraulic parameters (i.e., $K$ and $S_{\mathrm{s}}$ ) of geological units; (2) investigate the impact of different geological conceptualizations on the performance of groundwater model calibration and validation; and (3) explore the importance of geological data in improving the results of HT analysis at a large-scale field site.

Section "Description of groundwater models", fifth and sixth paragraphs should read:

In case 1 (the five-layer uni model), the initial hydraulic parameters for all layers are set to a uniform value. Specifically, the initial $K$ value for calibrating the five-layer geological model was set as $6.00 \times 10^{-5} \mathrm{~m} / \mathrm{s}$ with a minimum bound of $1.00 \times$ $10^{-9} \mathrm{~m} / \mathrm{s}$ and a maximum bound of $0.01 \mathrm{~m} / \mathrm{s}$. Likewise, the initial $S_{\mathrm{s}}$ value was set as $0.0006 \mathrm{~m}^{-1}$ with a minimum bound of $1.0 \times 10^{-8} \mathrm{~m}^{-1}$ and a maximum bound of $0.1 \mathrm{~m}^{-1}$. Since most observation points are located in AFB2, it is essential to set appropriate initial $K$ and $S_{\mathrm{s}}$ values for the other layers in order to increase the computational efficiency and reliability of results. Doherty (2005) stated that the properly selected initial parameter values will not only increase the optimization efficiency, but also transfer a highly nonlinear model to a reasonably linear model through parameter transformation. In particular, the predominant materials for each layer were identified and used to assign initial $K$ and $S_{\mathrm{s}}$ values, as shown in Table S2 of the ESM. The corresponding $K$ and $S_{\mathrm{s}}$ values for each material were based on Martin and Frind (1998), provided as Table S4 of the ESM. The representative values were identified by Martin and Frind (1998) from the literature and also calibrated based on previous pumping and slug test results at the same wellfield site. Since there are several water-supply wells located within the ATB3 unit of the Waterloo model, and in order to increase the computational efficiency, the initial $K$ and $S_{\mathrm{s}}$ values were set the same as AFB2 of the Waterloo model.

Four geological models with appropriate initial $K$ and $S_{\mathrm{s}}$ values for each layer were calibrated and validated as cases $2-5$. The minimum and maximum bounds of the estimated parameters in cases 2-5 are set the same as in case 1 .

Section "Model validation results", first paragraph should read:

The performances of different models in their ability to predict drawdown at monitoring wells were evaluated using the pumping/injection records from July to December, 2013. Simulated drawdowns are compared with corresponding observed drawdowns to provide quantitative evaluation. Similar to the calibration results, the validation results for four geological models with appropriate initial $K$ and $S_{\mathrm{s}}$ values (cases 2-5) are similar in their overall shape in terms of the point distribution and the slope of the fit lines, while the five-layer uni (uniform) model in case 1 yields the worst results with biased prediction. Section "Model validation results", third paragraph should read: While the validation scatterplots show great similarity among individual models (cases 2-5), the simulated and observed drawdowns for each observation well are provided (see Figs. S1 and $\mathrm{S} 2$ in the ESM) to better examine the performance of calibrated models in predicting drawdowns. The calibrated models for cases $2-5$ can generally capture the drawdown curves for most observation wells, while larger differences are mainly shown for manually measured observation wells. There are not much data available from these wells for model calibration, and some of these wells are located in deeper layers (e.g., AFC1) without reliable estimates of hydraulic parameters. Cases $2-5$ share more similarities compared with case 1 , while the five-layer uni model (case 1) and the five-layer geological model (case 2) can better capture the rapid changes in water levels. The reason behind this may be the higher $\mathrm{K}$ values for AT2 and the merging of AFB1, ATB2 and AFB2 into AF1, which resulted in higher-K pathways between the water-supply wells and observation boreholes. Another reason may be the use of an averaged daily schedule of pumping/injection rates, when in reality, the rates vary more abruptly and can impact water level records and corresponding parameter estimates (Luo et al. 2020).

2) The headings "Model 1. 5-layer geological mode" should be "Model 1. 5-layer geological model" and "Model 3. Waterloo and model 4. regional models" should be "Model 3. Waterloo and Model 4. Regional models".

3) All "regional model" should be "Regional model" in the text and captions (Figs. 4-10).

The original article has been corrected.

Publisher's note Springer Nature remains neutral with regard to jurisdictional claims in published maps and institutional affiliations. 